\title{
Extracting Stay Regions from UWB Indoor Trajectory and its Evaluation
}

\author{
Tessai Hayama *, Hiroki Takahashi *, Kazuya Nagatomo *
}

\begin{abstract}
Location-based technology is key for ubiquitous society. To enhance the location-based services, recognizing the places and the patterns where a person and an object have visited in addition to geographical locations is needed. Although some researchers have developed methods that extract outdoor stay regions from GPS trajectories to recognize the visiting places, there is no technology that recognizes stay regions, such as spatial location in a living house and an office building, from indoor trajectories. Technology for extracting indoor stay regions is required to achieve more intelligent indoor-location-based services, such as smart-home and smart-office. Therefore, we developed a method which extracts stay regions from an ultra-wideband (UWB) indoor trajectory. An UWB indoor positioning technology provides location information with a few-tens-of-centimeters error. Our developed method was evaluated comparing with conventional methods.
\end{abstract}

Keywords: indoor stay-region, trajectory mining, position-information clustering

\section{Introduction}

Location-based technology, such as using GPS and Wi-Fi, is key for ubiquitous society. To recognize spatial positions of a user, location-aware services which give information based on the user's location are performed. The location-based technologies have been adopted in many situations, such as product management in a factory, security by tracking a child's location, and transport navigation. To achieve the advances of the location-aware service, the system has to recognize spatial positions where a user has visited as symbolic place and infer the pattern of moving between the places so that the location-aware service can predict user's behavior and become more personalized.

Currently, location-based technologies are separated into indoor and outdoor use. The location-based technologies for outdoor are performed by GPS, which has already been common. On the other hand, the location-based technology for indoor location-aware service has not been standardized yet and so, some researchers are developing it using some kinds of sensor technologies, such as Wi-Fi[14], Bluetooth[15], and RFID[16]. Each of the location-based technology has both advantage and disadvantage based on the scope and

${ }^{*}$ Nagaoka University of Technology, Niigata, Japan 
accuracy of detecting the position of an object; i.e., Wi-Fi and Bluetooth have a larger scope of the detection than RFID, but recognize an object in accuracy with a-few-meter error and RFID recognizes in-small-area objects in high accuracy. Thus, the location-based technology to be adopted has to be chosen based on the requirement of the location-aware service.

The purpose of our research project is to develop technologies supporting activities that a person performs in an indoor space, such as smart-home, smart-factory, and smartoffice. For detecting such kinds of indoor human behaviors, an indoor positioning technology should be chosen based on the targeted room space and the detection accuracy with which the kinds of human activities can be identified. Thus, this paper presents a method for identifying places where a user has visited in indoor space by using wireless devices. Another purpose of our project is to develop an indoor positioning technology without prior knowledge about the place and persons' behavior. In recent years, an ultra-wideband (UWB) has been given attention to achieve indoor positioning systems as new wireless technology. An UWB indoor positioning system has features as follows; scope which can be detected by the UWB sensors is within less than $20 \mathrm{~m}$, an average accuracy in which an object is detected by using the UWB sensors is around $20 \mathrm{~cm}$, power consumption of UWB sensor is low, and UWB signal is not obscured by a wall and an obstacle[18]. For positioning using Wi-Fi and Bluetooth signals, location fingerprinting is commonly used; for positioning using UWB signals, trilateration is commonly used, making system-setting easier than the location fingerprinting.

Some researchers have studied on a positioning system of using UWB sensors and location-aware service based on the positioning system. For example, Duru et al. [5] investigated position-measuring algorithms for tracking an object with UWB sensors. Cai et al.[4] developed an ensemble learning particle swarm optimization algorithm for indoor target detection and showed that it almost correctly detected target positions in empty indoor environment. Although their method may be useful to identify each tag position, it is not evaluated for environment with Non-Line-of-Sight condition and multi-path problem yet, and the purpose of the algorithm is not to detect the stay regions of the target. Alhadhrami et al.[1] used a UWB positioning system to develop a voice-guide service for a blind person. The service gives information showing the user's surrounding state and guides the direction to exit the room from the user's current position. Huo et al.[8] developed an augmented reality system which supports the discovery of the surrounding smart things and their locations through an augmented reality smart system. The previous studies of using UWB sensors have developed systems giving a service at current position of a UWB tag. To improve intelligence of indoor location-aware service, the location-based system has to recognize the user's positions detected by UWB sensors as symbolic place of the user's destination; so the system is able to infer daily routines and contextual behaviors by using user model made from the user's movement [10]. To achieve this, the use of position of interest (PoI) database for target indoor is the best approach; however, due to high cost such as human labor and time, making such kind of knowledge base for each indoor is difficult.

Therefore, we developed a method for extracting stay regions from UWB indoor trajectory. Our proposed method is based on the time-based clustering[9] which is an algorithm for extracting stay regions from GPS trajectry. We combined the time-based clustering algorithm with two factors, user's direction and location, to extract stay regions from indoor geospatial trajectory. Our developed method was evaluated and used to compare with conventional methods. The results showed our proposed method correctly extracts stay regions from indoor geospatial trajectory with easy parameter settings. 


\section{Approach}

The purpose of this study is to develop a method for extracting stay regions where a user has visited from UWB indoor trajectory made during the user's movement. This section shows settings of this study, introduction to the previous algorithms for extracting stay regions from GPS trajectory, and design policy of a method for extracting indoor stay regions, accordingly.

\subsection{Settings}

We define UWB trajectory data and a stay region which is extracted from the UWB trajectory data, and also show an indoor environment where the UWB trajectory data is made for this study.

To get positions of a user's indoor movement, an UWB tag is attached to the user and each UWB anchor placed at a different corner of the room detects the distance from the UWB tag by using the time-of-arrival method (TOA). Then, based on the distances between the UWB tag and each of the UWB anchors by a measuring method, such as trilateration, the spatial indoor-position of the user is calculated. The positions of the object are obtained with the timestamp at a higher rate of 10 per second.

An UWB tag position $p_{i}$ is defined as a tuple $\left(x_{i}, y_{i}, t_{i}\right) ; x_{i}$ and $y_{i}$ represent the $x$ and $y$ of two-dimensional coordinate at time $t_{i}$. UWB trajectory $T$ of UWB tag positions of a user is a set of $n$ temporarily increasing points; $T$ is defined as $\left\{p_{1}, p_{2}, \ldots, p_{n}\right\}$. UWB trajectory $T$ contains positions where a user has intentionally visited and positions during moving to another place from the place where a user stayed. A place where a user intentionally visited is defined as stay region which is a set of the consecutive positions where the user intentionally visited; Place is represented as follows;

$$
\text { Place }=\left\{p_{i}, p_{k}, \ldots, p_{n}\right\}, t_{i}<t_{k}<t_{n}, p_{i}, p_{k}, p_{n} \in T .
$$

An UWB positioning system makes UWB trajectory including outlier and missing position, due to the nature of indoor multipath propagation environment and environment with non-line-of-sight (NLOS) condition between positions of an anchor and a tag. The UWB localization system will dramatically degrade the performance of the conventional localization method by the multipath propagation and NLOS problems. Thus, a method for extracting stay regions from UWB trajectory inputs $T$ and then outputs Places by not exerting influence on such UWB errors.

\subsection{Previous Algorithms for Extracting Stay Regions from GPS Outdoor Tra- jectory}

Although there has not been an algorithm for extracting stay regions from UWB indoor trajectory yet, some researchers have developed algorithms for extracting stay regions from GPS outdoor trajectory. They have tackled the problem on GPS detection error within GPS trajectory. Thus, to develop an algorithm for extracting stay regions from UWB indoor trajectory, we take the previous algorithms for outdoor into account.

According to the literature[12], the algorithms for extracting stay regions from GPS outdoor trajectory are categorized into four types; density-based, rapid-based, time-based, and direction-based. The overview of the four is described as follows: 
- A density-based algorithm, which relies on a density-based notion of clusters is designed to discover the clusters and the noise in a spatial database[6]. The density within the areas of noise is lower than the density in any of the clusters. The algorithm requires only one input parameter to determine density-reachable points.

- A rapid-based algorithm, which extracts low-speed segments, where the instantaneous speed between two consecutive points is lower than that in other parts of the same trajectory corresponds to interesting places[13]. The other algorithm[3], which assigns stop to PoIs in a given PoI database, estimates the location and time of a user and then ranks the PoIs using a line-segment-intersection-based approach.

- A time-based algorithm is based on clustering the locations along the time axis. The measured new-location is compared with previous locations and then, if the new location is moving away from the previous locations, the new location is considered to belong to a different cluster[9].

- A direction-based algorithm, which buckets speed and acceleration to identify all those segments of a GPS trajectory and then extracts segments of the user's positions with a distribution of bearing change and standard deviation of each segment[2]. The main idea of the algorithm is that the bearing is random in nature when a user is at a point-like or an extended place.

\subsection{Design Policy}

Normally, due to the user's activity in small space, moving distance and time spending for stay are much shorter indoor than outdoor. If the previous algorithms for GPS outdoor trajectory are adopted to extract stay regions from an UWB indoor trajectory, it is difficult to extract correctly the indoor stay regions. For example, the density-based algorithm is likely not to extract any position in each correct stay region but extract positions on an aisle which a user often go through. The density-based algorithm is not able to judge if the density of positions during certain short time is at stay region or not. Since the moving distance of the user is often too short indoor, the rapid-based algorithm may not judge the differences between the moving position and stay position of a user. On the other hand, the time-based algorithm and direction-based algorithm have the possibility to correctly extract stay regions from an UWB indoor trajectory. For example, the time-based algorithm selects consecutive positions of a user during a certain time to extract stay region where the user visited; however, it is difficult to set the time parameter because of requesting the sensitive parameter in case of a user's movement for picking something up. The directionbased algorithm can detect the clustered positions where a user has stayed indoor as well as outdoor; however, it is difficult to determine the parameter of the direction randomness because of user's little movement indoor.

User's indoor location is one of the useful factors used to extract stay regions from the indoor trajectory. In many cases, there are furniture and equipment along the wall at the room, such as home and office. If a user moves toward the wall and then stays near the wall, the user may have a destination to the furniture or equipment along the wall. Therefore, based on the three factors (time, direction, and location), we develop an algorithm which extracts stay regions from UWB indoor trajectory. 


\section{Proposed Method}

We developed a method for extracting stay regions from UWB indoor trajectory based on the design policy which is described in section II.C. The developed method firstly chooses three anchors among some anchors at the indoor space for estimating the position of a tag to deal with the NLOS condition and multipath propagation problems. The anchors are chosen by the smallest-three standard deviation for $\mathrm{N}$ more-recent-distance values between the anchor and tag. Then, the developed method extracts stay regions by our proposed algorithm in which the time-based clustering algorithm[9] is combined with two factors; direction and location. The pseudo code of the developed method is shown in Figure 1.

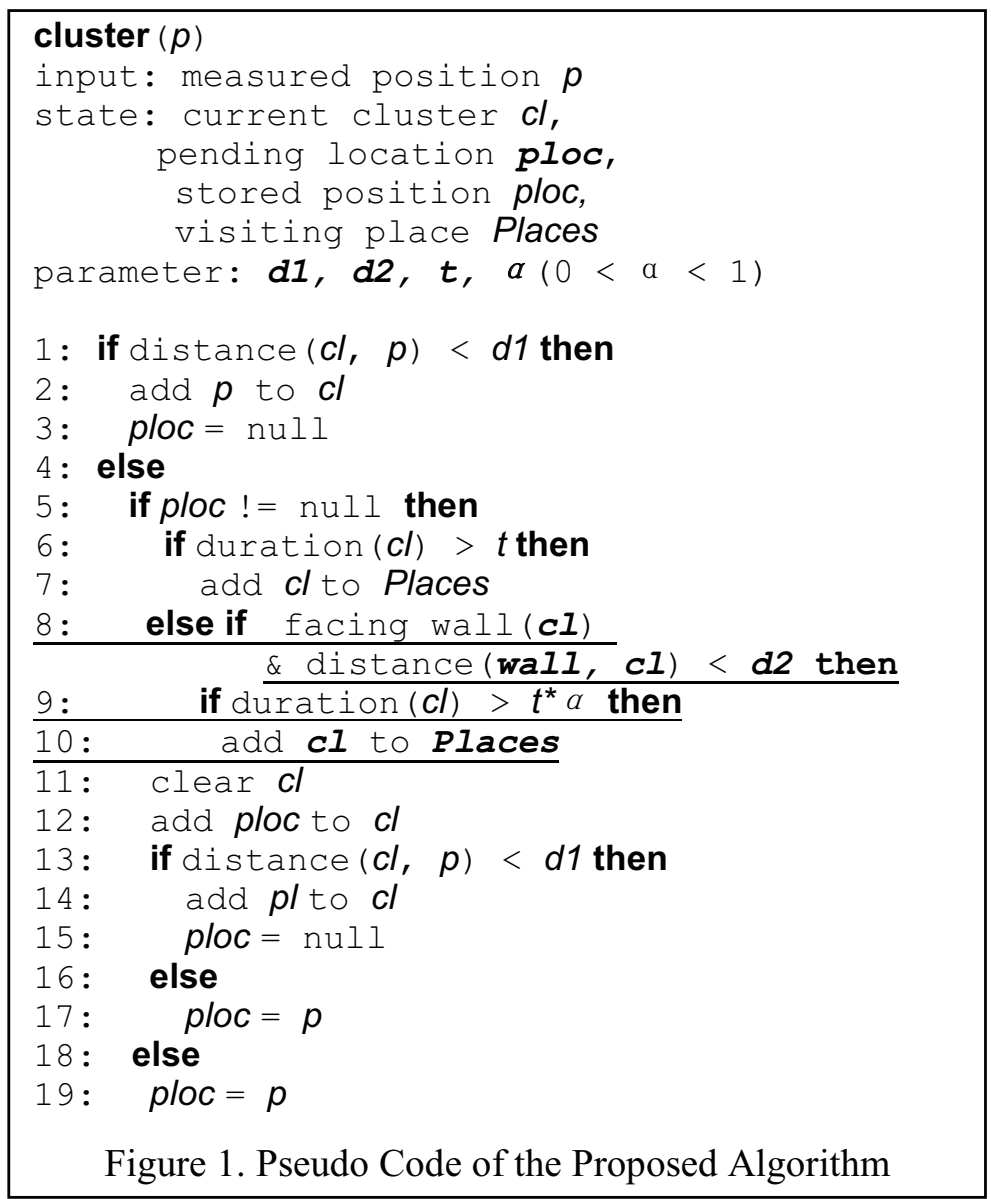

Figure 1: Pseudo Code of the Proposed Algorithm.

Based on utilizing a user's direction and location, the line 8-10 of the pseudo code of the proposed algorithm shows a part of the clustering processing. The part of the clustering process means that stay region during shorter time than the threshold $t * \alpha$ is added into the list of the user's visited places, Places, if many user's positions within the cluster $\mathrm{cl}$ are directed to the wall and the centroid of $c l$ is less than parameter $d 2$ far from the wall. Therefore, a cluster in which many positions are directed to the wall and are located near the wall is likely to be recognized as stay region, even if the cluster is in short time stay.

The proposed algorithm contains two types of parameters which are concerned with distance and time. The distance parameter $d 1$ and $d 2$ represent the distance between a 
position and the position next to it for making a cluster and distance between a position and wall near it for giving the advantage of the location, respectively. Both distance parameters are set to be about the same values as an average UWB error. The time parameter $t$ repre-sents stay-duration to recognize a cluster in a stay region. The parameter $t$ is set to be a few seconds to detect simple human's indoor-behavior.

After finishing the clustering process, each cluster is combined with the other cluster if the distance between the two clusters is the distance parameter $d 3$. The parameter $d 3$ is set to be twice of an average UWB error, although the density-based algorithm[6] for GPS outdoor trajectory set the distance parameter $d 3$ into half of $d 1$.

\section{Evaluation}

\subsection{Procedures}

By comparing our proposed method to the conventional method, we confirmed the effects of our proposed method with UWB trajectory data.

The UWB trajectory data which was used for the evaluation was made in the room, our laboratory, as shown in Fig. 2 by six persons who were attached with a UWB tag. The persons run on one-hour schedules which were made by combining the following items; 1) working on their own desk, 2) picking up a book on the shelf, 3) picking up a device on the shelf, 4) picking up a copy of a document on the printer, 5) making tea nearby the home-electric appliance shelf, 6) relaxing on the sofa chair, and 7) looking outside from the window. More than 70,000 UWB positions with timestamp were obtained as the UWB trajectory data of the experiment, which included more than 100 UWB missing positions and 44 stay regions which the person intentionally visited.

Four UWB anchors were set to on $2250 \mathrm{~mm}$ height at each corner of the room. There were 12 desks and a PC on each of the steel desk, sofa chairs, two steel bookshelves, two steel equipment shelves, a printer, some home-electric appliances, four wifi and so on. As UWB sensor and device to communicate with the dwm1001 sensor module in the experiment, the DWM1001 sensor module ${ }^{1}$ and Raspberry $\mathrm{pi}^{2}$ were used, respectively. More than three persons worked in the room during the experiment. In the experimental environment, we investigated the detection accuracy of a UWB tag position. As shown in Figure 2, the UWB-position detection accuracy was measured one-hundred times for 12 positions of the room. The positions of all tags were in NLOS conditions against the one of the anchors. TABLE 1 shows the results of the UWB-position detection accuracy. In the results, the average detection error on $\mathrm{x}$-axis and $\mathrm{y}$-axis were $183.93 \mathrm{~mm}$ and $159.91 \mathrm{~mm}$, respectively, and maximum detection error of the UWB-position on $\mathrm{x}$-axis and $\mathrm{y}$-axis were $487.74 \mathrm{~mm}$ and $482.24 \mathrm{~mm}$, respectively. Since the maximum error of the UWB-position detection is about the same wide as a usual adult body, detecting the person's position by UWB sensors is allowed for tracking the person's behavior in the experiment. In the measuring positions with id 7,8 and 11, the accuracies of the UWB-detection were lower than the others, since the average of the UWB-detection error with the ids were larger than $200 \mathrm{~mm}$ on $\mathrm{x}$-axis and y-axis. We found that the reason of the low accuracy of the UWBdetection at their positions was the NLOS condition for more than two anchors and the tag.

\footnotetext{
${ }^{1}$ https://www.decawave.com/product/dwm1001-development-board/

${ }^{2}$ https://www.raspberrypi.org/
} 


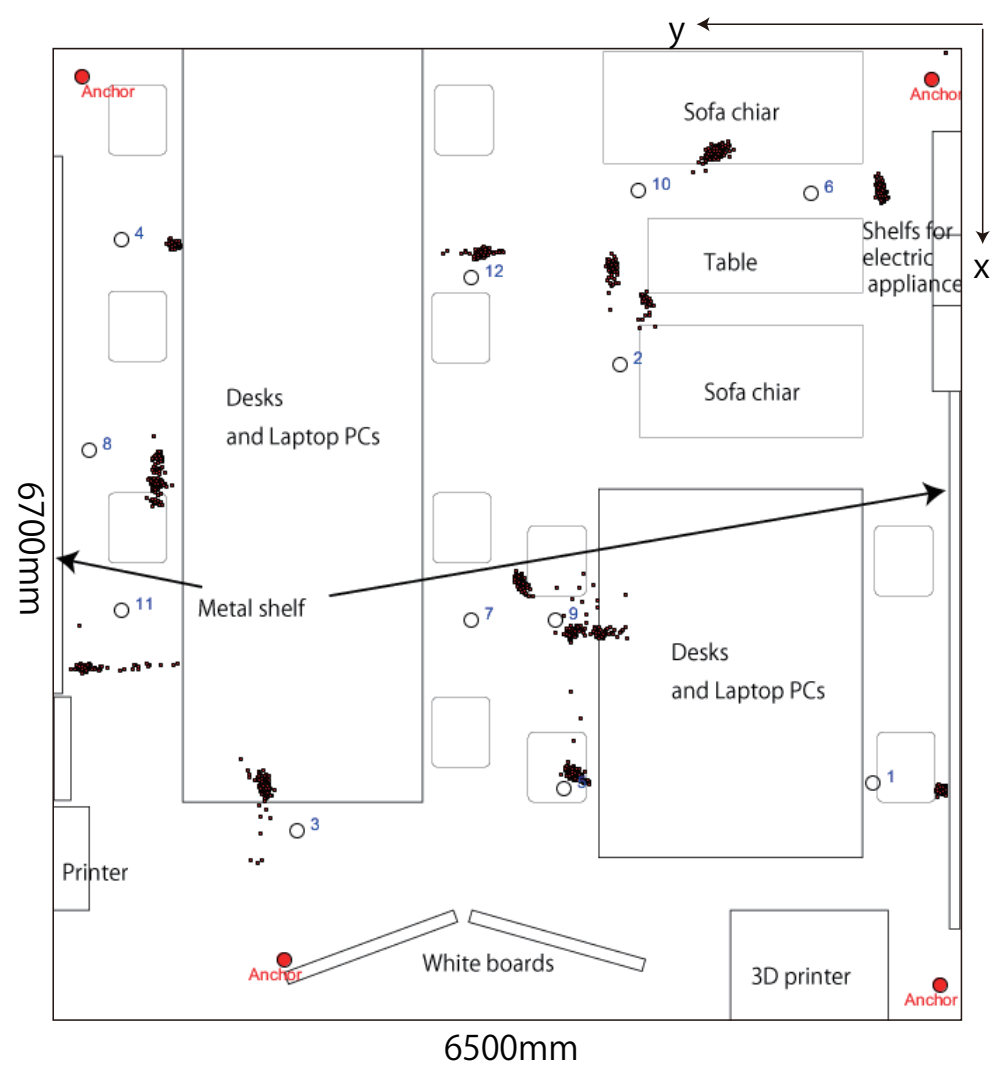

Figure 2: Layout of a room where the experiment was performed. (number represent position where the UWB sensor was evaluated and it's id. )

Table 1: Results of UWB-position detection accuracy in the investigation.

\begin{tabular}{c|rrrr|rrrr}
\hline $\begin{array}{c}\text { Measuring } \\
\text { position }\end{array}$ & \multicolumn{3}{|c}{ Error (mm) on x-axis } & \multicolumn{4}{c}{ Error (mm) on y-axis } \\
\hline 1 & 72.21 & 46.25 & 264.97 & 1.81 & 219.83 & 71.42 & 482.24 & 51.03 \\
2 & 265.45 & 33.15 & 321.78 & 38.69 & 93.31 & 59.81 & 362.13 & 3.16 \\
3 & 43.79 & 42.78 & 244.03 & 0.86 & 188.70 & 67.23 & 466.89 & 68.91 \\
4 & 39.32 & 19.39 & 85.60 & 0.05 & 58.95 & 27.78 & 161.90 & 9.23 \\
5 & 56.20 & 41.87 & 424.56 & 11.25 & 67.93 & 35.83 & 222.01 & 14.06 \\
6 & 307.82 & 176.84 & 487.74 & 50.78 & 64.31 & 18.80 & 113.93 & 23.53 \\
7 & 268.61 & 38.24 & 352.76 & 171.68 & 213.70 & 35.36 & 294.16 & 138.83 \\
8 & 200.82 & 113.34 & 399.99 & 10.54 & 218.92 & 26.54 & 284.32 & 119.69 \\
9 & 99.31 & 62.45 & 277.30 & 2.72 & 275.09 & 69.02 & 354.20 & 3.13 \\
10 & 270.67 & 34.12 & 353.73 & 141.57 & 95.70 & 16.86 & 141.12 & 63.87 \\
11 & 406.74 & 31.09 & 440.14 & 126.12 & 388.74 & 12.62 & 390.00 & 263.80 \\
12 & 176.24 & 16.21 & 212.29 & 131.97 & 33.72 & 13.66 & 59.28 & 0.68 \\
\hline All & 183.93 & 54.64 & 487.74 & 0.05 & 159.91 & 37.91 & 482.24 & 0.68 \\
\hline
\end{tabular}


The proposed method was compared with the algorithm for GPS stay-region extraction, the time-based algorithm[9], by precision, recall and f-measure scores of the extracted stayregions within the correct stay-regions. The algorithms were adapted with parameter tuning to the UWB indoor trajectory.

\subsection{Results and discussions}

First, we show the results of parameters which were investigated for the algorithms by using a sample data set of UWB trajectory with 20 stay-regions; the data set was not included in the experimental data to investigate the accuracy[7]. Then, we show the accuracy of the algorithms in extracting the stay regions from the UWB trajectory based on the investigated parameters.

\subsubsection{Investigation results on parameters of the algorithms}

Figures 3 and 4 show numbers of the clusters (stay regions) found in parameters of time threshold and different distance by the proposed method and the time-based algorithm, respectively.

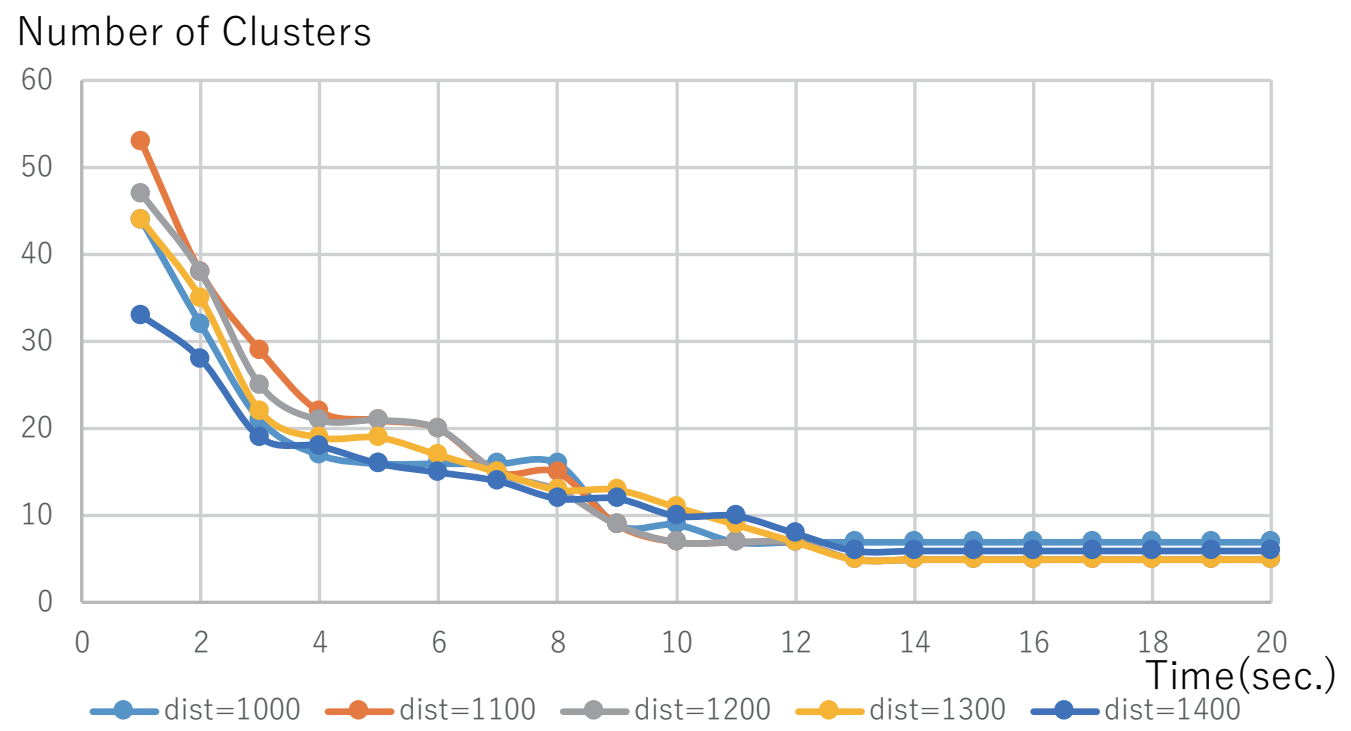

Figure 3: Number of the clusters (stay regions) detected in parameters of time threshold and different distance by the proposed method.

The proposed method had a similar tendency of the parameter settings to the time-based algorithm. In the parameter of time threshold, both algorithms detected a close number of the correct stay regions, 20, by setting 3-13 seconds to the parameter. The proposed method had more parameters of the time threshold than the time-based algorithm to detect all of the correct stay regions. In the distance parameter, both algorithms detected the stay regions more correctly with the parameter setting of 1100,1200 , and $1300 \mathrm{~mm}$ than the parameter setting of $1000 \mathrm{~mm}$ or $1400 \mathrm{~mm}$; the tendency of which is stronger in the proposed method than the time-based algorithm. The distance parameter may indicate a normal walking speed of around $1200 \mathrm{~mm}$ per second. Therefore, the parameter settings of the proposed method are easier than the ones of the time-based algorithm. 


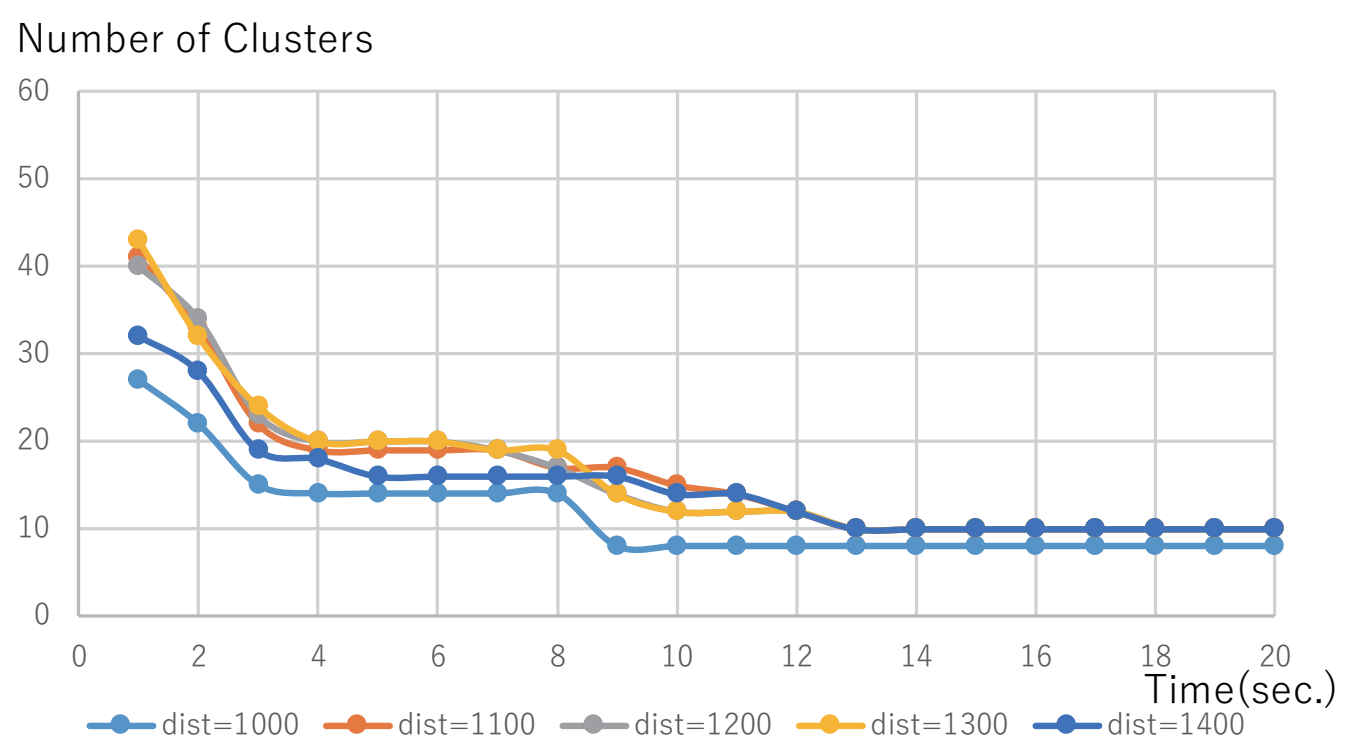

Figure 4: Number of the clusters (stay regions) detected in parameters of time threshold and different distance by the time-based algorithm[9].

\subsubsection{Results on stay-region extraction}

Figrures 5-7 show precision, recall, and f-measure scores of the proposed method and the time-based algorithm[9]. Based on the investigated parameters, the proposed algorithm and the time-based algorithm were set to more than 5 and 1100-1300, respectively as time threshold parameter and distance parameter.

In both scores of the precision and the f-measure, the proposed method obtains better results over the investigated time than the time-based algorithm as shown in Fig. 5 and 7, respectively. In the recall score, the proposed method obtains better result in $25 \mathrm{~s}$ or more; however, the time-based algorithm obtains better result in less than $25 \mathrm{~s}$ as shown in Fig. 6. Detecting accurate stay-regions is required to recognize human behaviors, meaning that the precision is more important than the recall. Therefore, the proposed method is useful to extract the stay regions from the UWB indoor trajectory with easy parameter settings.

Fig. 8 and 9 show the example of the UWB trajectory with the person and the results of extracting stay-regions from the UWB trajectory by the proposed method. In the example, the UWB trajectory while moving to another place was removed by the proposed method. However, it couldn't recognize some stay-regions in quickly picking up something looking outside from the window. When the person sat at his desk, it was difficult for the current UWB localization technology to get more accurate positions than the other since the tag was under the desk. Although the UWB location technology can detect such stay-region in the experiment, it is required to correct the positions which the UWB location technology detects in such the condition.

\section{Conclusion}

For location-aware service in ubiquitous society, recognizing symbolic places where a person has visited is becoming a more important technology in addition to recognizing a per- 


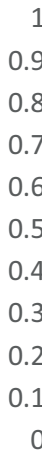

0.6

0.5

0.4

$$
\begin{aligned}
& \text { s. } \\
& \begin{array}{llllllllllllllllllll}
5 & 10 & 15 & 20 & 25 & 30 & 35 & 40 & 45 & 50 & 55 & 60 & 65 & 70 & 75 & 80 & 85 & 90 & 95 & 100 \\
\text { sec. }
\end{array}
\end{aligned}
$$

- - - Time-based clustering(d=1100) - - - Time-based clustering(d=1200)

- - - Time-based clustering $(d=1300) \longrightarrow$ The proposed method $(d=1100)$

The proposed method $(\mathrm{d}=1200)$ The proposed method $(\mathrm{d}=1300)$

Figure 5: Precision of the proposed method and the time-based algorithm[9].

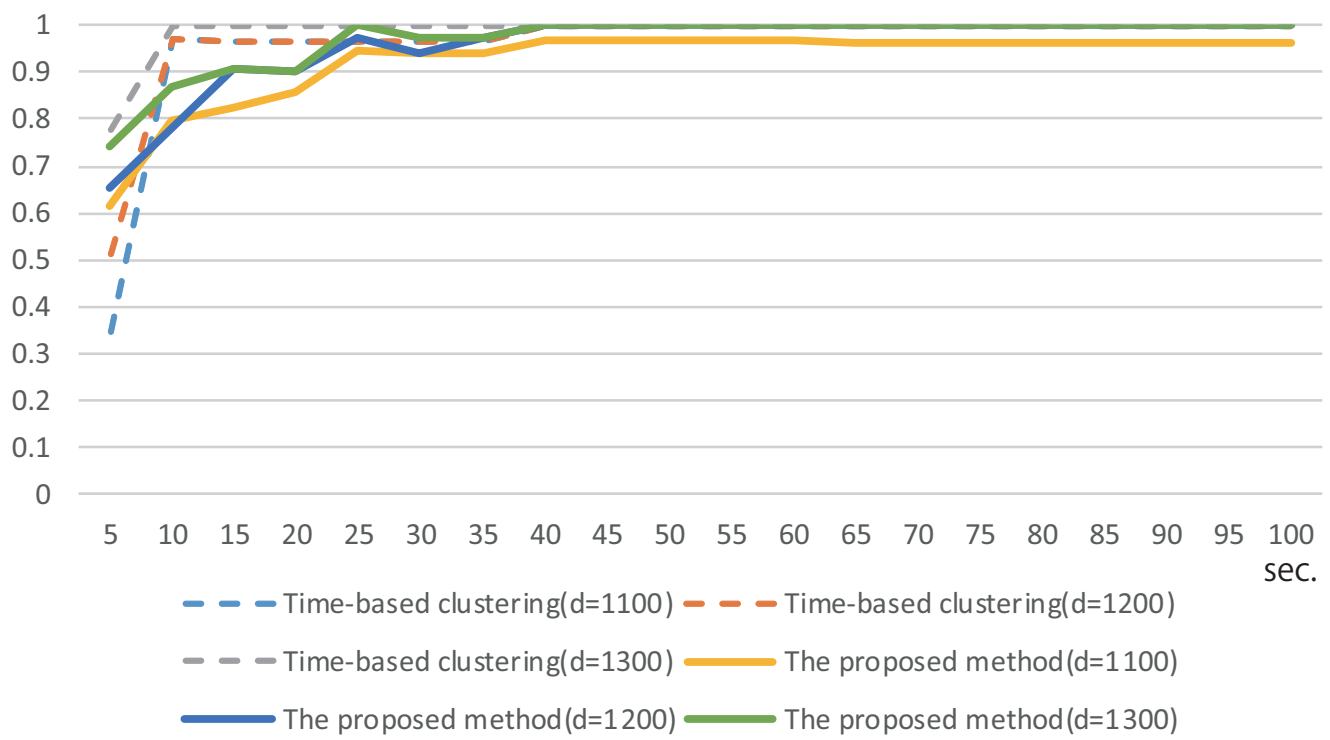

Figure 6: Recall of the proposed method and the time-based algorithm[9]. 


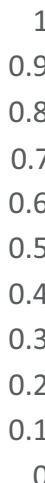

$$
\begin{aligned}
& 0.8 \\
& 0.7 \\
& 0.6
\end{aligned}
$$$$
0.7
$$

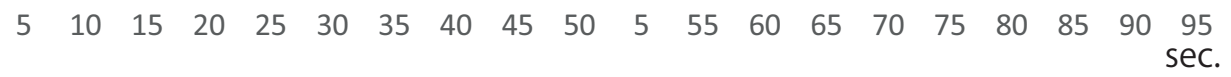

- - Time-based clustering $(\mathrm{d}=1100)$ - - - Time-based clustering $(\mathrm{d}=1200)$

- - Time-based clustering $(\mathrm{d}=1300) \longrightarrow$ The proposed method( $\mathrm{d}=1100)$

The proposed method $(d=1200)$ The proposed method $(d=1300)$

Figure 7: F-measure of the proposed method and the time-based algorithm[9].

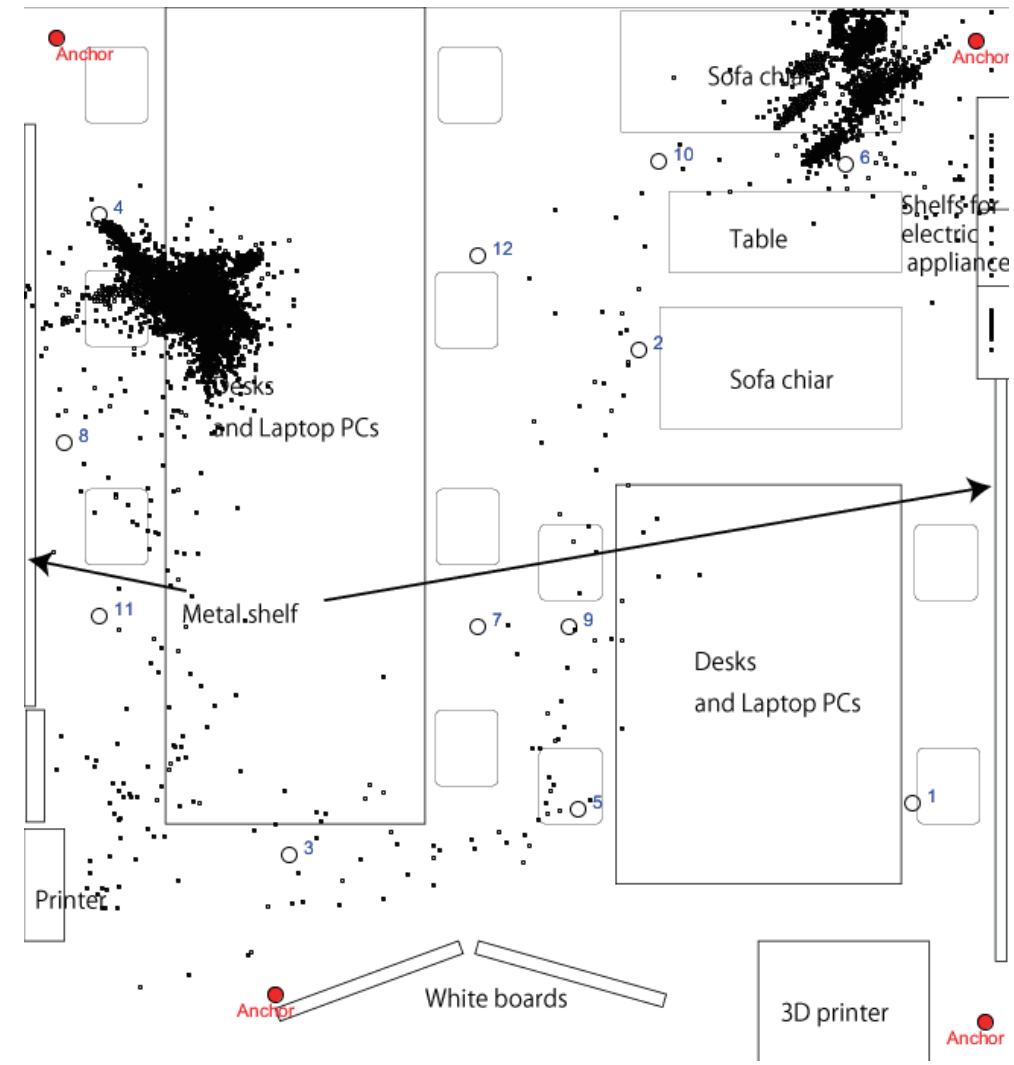

Figure 8: Example of UWB trajectory obtained in the experiment. 


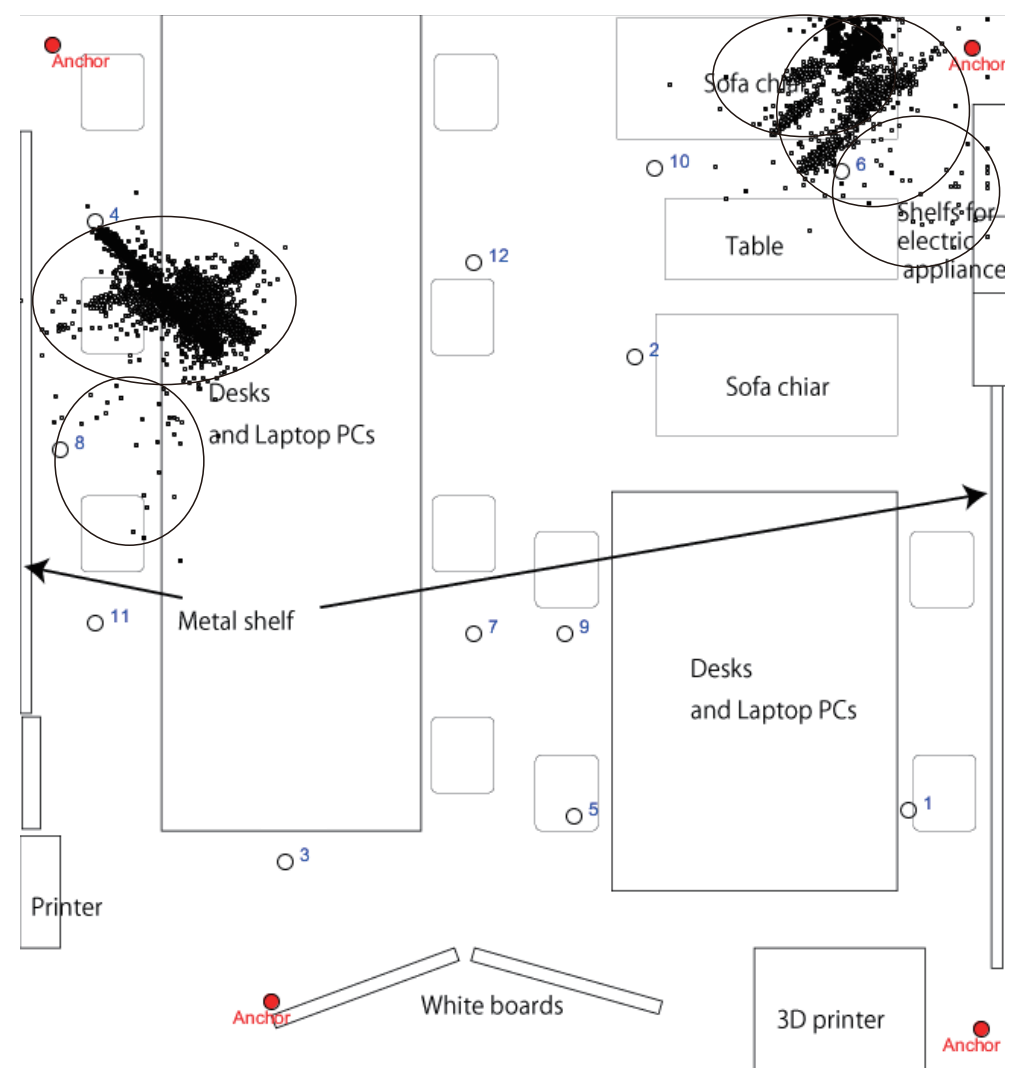

Figure 9: Results of extracting stay-regions from UWB trajectory by the proposed method (Circles indicate the extracted stay-regions).

son's position. In this study, we developed a method for extracting stay regions from the UWB indoor trajectory. The developed method is based on the time-based algorithm which is combined with the two factors (user's location and direction) for indoor. To confirm that the developed method worked well, we established an UWB indoor environment to get the UWB trajectory data and compared to the conventional method by the trajectory data. The evaluation results showed the proposed method correctly extracts stay regions from UWB indoor trajectory with easy parameter settings.

In our future works, we will improve the UWB positioning detection accuracy and evaluate the proposed method in practice. And also we are going to develop some intelligent services by using the indoor positioning system.

\section{Acknowledgment}

This work was supported by JSPS KAKENHI Grant Number 19K12264.

\section{References}

[1] S. Alhadhrami, A. Alnafessah, M. Al-Ammar, A. Alarifi, H. Al-khalifa, and M. Alsaleh: UWB Indoor Tracking System for Visually Impaired People. Procs. the 13th 
International Conference on Advances in Mobile Computing and Multimedia (MoMM 2015), pp.54-62 (2015).

[2] T. Bhattacharya, L. Kulik, and J. Bailey: Extracting significant places from mobile user GPS trajectories: a bearing change based approach. In SIGSPATIAL/GIS, pp. 398-401 (2012).

[3] T. Bhattacharya, L. Kulik, and J. Bailey: Automatically recognizing places of interest from unreliable GPS data using spatio-temporal density estimation and line intersections. Pervasive Mob. Comput. 19 , pp. 86-107 (2015).

[4] X. Cai, L. Ye, and Qin Zhang:Ensemble learning particle swarm optimization for realtime UWB indoor localization. EURASIP Journal on Wireless Communications and Networking (2018).

[5] A. Duru, E. Sehirli, and Idris Kabalc: Ultra-Wideband Positioning System Using TWR and Lateration Methods. Procs.the Fourth International Conference on Engineering \& MIS 2018 (ICEMIS '18). Article 58-61 (2018).

[6] M. Ester, H. Kriegel, J. Sander, and X. Xu: A density-based algorithm for discovering clusters in large spatial databases with noise. Procs. the Second International Conference on Knowledge Discovery and Data Mining (KDD'96), AAAI Press pp.226231(1996).

[7] T. Hayama, T. Nariai and K. Nagatomo: Extracting Stay Regions from UWB Indoor Trajectory. Procs. the Eighth International Congress on Advanced Applied Informatics (2019).

[8] K. Huo, Y. Cao, S. H. Yoon, Z. Xu, G. Chen, and K. Ramani: Scenariot: Spatially Mapping Smart Things Within Augmented Reality Scenes. Procs the 2018 CHI Conference on Human Factors in Computing Systems (CHI '18), pp. 219-231 (2018)

[9] J. H. Kang, W. Welbourne, B.Stewart, and G. Borriello: Extracting places from traces of locations. Procs. the 2nd ACM international workshop on Wireless mobile applications and services on WLAN hotspots (WMASH '04), pp.110-118 (2004).

[10] L. Liao, D. J. Patterson, D. Fox, and H. Kautz: Learning and inferring transportation routines. Artif. Intell. 171, pp.311-331 (2007).

[11] Y. Ma, N. Selby, M. Singh, and F. Adib: Fine-grained RFID Localization via Ultrawideband Emulation. Procs. the SIGCOMM Posters and Demos (SIGCOMM Posters and Demos '17). pp.116-118 (2017).

[12] K. Nishida, H. Toda, and Y. Koike: Extracting Arbitrary-shaped Stay Regions from Geospatial Trajectories with Outliers and Missing Points. Procs. the 8th ACM SIGSPATIAL International Workshop on Computational Transportation Science (IWCTS'15), pp. 1-6(2015).

[13] A. T. Palma, V. Bogorny, B. Kuijpers, and L. O. Alvares: A clustering-based approach for discovering interesting places in trajectories. Procs. the 2008 ACM symposium on Applied computing (SAC '08), pp.863-868(2008). 
[14] J. Wang and D. Katabi. Dude, where's my card?: R d positioning that works with multipath and non-line of sight. In ACM SIGCOMM Computer Communication Review, volume 43, pp. 51-62 (2013).

[15] Y. Wang, X. Yang, Y. Zhao, Y. Liu, and L. Cuthbert.Bluetooth positioning using rssi and triangulation methods. In Consumer Communications and Networking Conference (CCNC), 2013 IEEE, pp. 837-842(2013).

[16] D. Weng, Y. Liu, Y. Wang, and L. Wu: Study on an indoor tracking system based on primary and assistant infrared markers. In Computer-Aided Design and Computer Graphics, 2007 10th IEEE International Conference on, pp. 377-382 (2007).

[17] Y. Zheng, L. Zhang, X. Xie, and W. Ma: Mining interesting locations and travel sequences from GPS trajectories. Procs. the 18th international conference on World wide web (WWW '09). pp.791-800 (2009)

[18] F. Zafari, A. Gkelias, and K. K. Leung: A Survey of Indoor Localization Systems and Technologies. IEEE Communications Surveys \& Tutorials, Vol.21, pp.2568-2599 (2017). 